\title{
A Code to Compute the Action-Angle Transformation for a Particle in an Arbitrary Potential Well ${ }^{*}$

\author{
J. Scott Berg and Robert. L. Warnock \\ SCAN-9507227 \\ Stanford Linear Accelerator Center; Stanford University; Stanford, CA 94309

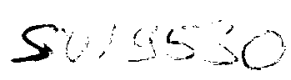

\begin{abstract}
For a Vlasov treatment of longitudinal stability under an arbitrary wake field, with the solution of the Haissinski equation as the unperturbed distribution, it is important to have the action-angle transformation for the distorted potential well in a convenient form. We have written a code that gives the transformation $q, p \rightarrow J, \phi$, with $q(J, \phi)$ as a Fourier series in $\phi$, the Fourier coefficients and the Hamiltonian $H(J)$ being spline functions of $J$ in $C^{2}$ (having continuous second derivatives).
\end{abstract}

\section{The Canonical Transformation}

We suppose that the Hamiltonian has the form

$$
H=\frac{p^{2}}{2}+V(q)
$$

where $V(q)$ is a potential well with continuous derivative. We discuss only values of the constant $H$ such that the motion consists entirely of oscillations between two turning points at which $p=0$. We denote the turning points by $q_{0}$ and $q_{1}$, with $q_{0}<q_{1}$, and exclude values of $H$ for which either $V^{\prime}\left(q_{0}\right)$ or $V^{\prime}\left(q_{1}\right)$ is zero. We define

$$
p(q, H)= \pm \sqrt{2[H-V(q)]}
$$

where $p>0$ as $q$ moves from $q_{0}$ to $q_{1}$, and $p<0$ as it returns from $q_{1}$ to $q_{0}$. The action integral, which extends over a full period of the motion, is

$$
J(H)=\frac{1}{2 \pi} \oint p(q, H) d q=\frac{1}{\pi} \int_{q_{0}}^{q_{1}} p(q, H) d q .
$$

Thanks to our assumption that $V^{\prime}\left(q_{i}\right) \neq 0$, there is a welldefined inverse function $H(J)$.

Hamilton's equations imply that $p=d q / d t$. If $t=0$ at $q=q_{1}$, the time $t$ for displacement $q$ is

$$
t=\int_{q_{1}}^{q} \frac{d q^{\prime}}{p\left(q^{\prime}, H\right)}
$$

where the integration path is understood to follow all oscillations that occur by time $t: q_{1} \longrightarrow q_{0} \longrightarrow q_{1} \longrightarrow \cdots \longrightarrow$ $q(t)$. Since $H$ depends only on $J$, Hamilton's equations in action-angle variables give $\Phi=\Phi_{0}+H^{\prime}(J) t$. Choosing $\Phi\left(q_{1}\right)=0$, we have

$$
\Phi(q, H)=H^{\prime}(J(H)) \int_{q_{1}}^{q} \frac{d q^{\prime}}{p\left(q^{\prime}, H\right)}
$$

* Work supported by the Department Energy, contract DE-ACO376 SF00515.
We wish to find the functions $q(J, \Phi), p(J, \Phi)$, and $H(J)$ in a form that will be convenient for repeated and fast numerical evaluations, with $2 \pi$-periodicity in $\Phi$ guaranteed. We also want these functions to have continuous second derivatives in both variables. These requirements arise from an intended application in solutions of the Vlasov equation with Fokker-Plank term, as discussed below. A convenient expression of the functions is

$$
\begin{aligned}
& q(J, \Phi)=\sum_{m=0}^{\infty} q_{m}(J) \cos m \Phi \\
& p(J, \Phi)=\frac{\partial q(J, \Phi)}{\partial \Phi} H^{\prime}(J)
\end{aligned}
$$

with $q_{m}(J)$ and $H(J)$ expanded in terms of some $C^{2}$ basis functions $B_{k}(J)$ and $C_{k}(J)$ :

$$
\begin{aligned}
& q_{m}(J)=\sum_{k} q_{m k} B_{k}(J) \\
& H(J)=\sum_{k} h_{k} C_{k}(J)
\end{aligned}
$$

The formula (7) follows from the derivative of (5) with respect to $\Phi$, if we recall that $H$ is only a function of $J$ when written in action-angle coordinates.

If the series (6), (8), and (9) are truncated at a finite number of terms, the resulting transformation $J, \Phi \rightarrow q, p$ will not be precisely canonical (i.e., symplectic). A measure of symplecticity is the agreement of $p$ as given in (7) with

$$
p(J, \Phi)= \pm \sqrt{2[H(J)-V(q(J, \Phi))]} .
$$

If $p$ is given by (10), a calculation of the Poisson bracket yields

$$
[q, p]=\frac{1}{p} \frac{\partial q}{\partial \Phi} \frac{d H}{d J} .
$$

Thus, if $p$ from (7) agrees with $p$ from (10), we have a canonical transformation, since $[q, p]=1$. With a moderate number of terms in the series (6), (8), and (9), the transformation can be made to satisfy the canonical condition with sufficient precision for our purposes.

\section{The Primary Integrations}

We first evaluate the integrals (3) and (5) on a regular mesh in $H:\left\{H_{i} \mid i=1, \ldots, K\right\}$. The turning points $q_{0}\left(H_{i}\right)$ and $q_{1}\left(H_{i}\right)$ are easily found by a Newton iteration. The factor $H^{\prime}\left(J\left(H_{i}\right)\right)$ is defined at each $i$ by

$$
\pi=H^{\prime}\left(J\left(H_{i}\right)\right) \int_{g_{1}}^{q_{0}} \frac{d q}{p\left(q, H_{i}\right)} .
$$


For numerical integration, it is useful to change the variable to

$$
u=\cos ^{-1} \frac{q_{1}+q_{0}-2 q}{q_{0}-q_{1}} .
$$

The inverse of this transformation is

$$
q=\frac{q_{0}+q_{1}}{2}+\frac{q_{1}-q_{0}}{2} \cos u
$$

Then (5) becomes

$$
\Phi\left(q, H_{i}\right)=\frac{q_{1}-q_{0}}{2} H^{\prime}\left(J\left(H_{i}\right)\right) \int_{0}^{u(q)} \frac{\sin u^{\prime} d u^{\prime}}{\sqrt{2\left[H_{i}-V\left(q\left(u^{\prime}\right)\right)\right]}}
$$

The integrand is now free of singularities. For a nearly quadratic potential, $\Phi$ is close to $u$. The same change of variable is used to compute $J\left(H_{i}\right)$ by $(3)$.

Now $u=\pi$ corresponds to $q=q_{0}$. We divide the interval $[0, \pi]$ into $N$ intervals, and integrate by Simpson's rule [1]. The first and last intervals are treated by an open NewtonCotes formula [1], to avoid taking the limit of the integrand at the endpoints. We evaluate the integrand (15) for upper limit $u$ at all of the mesh points $u_{i}$. The value of $N$ is increased until the integral on $[0, \pi]$ converges to machine precision.

\section{Finding the Fourier Coefficients}

After the integrations, the angles $\Phi^{(j)}=\Phi\left(q\left(u_{j}\right), H_{i}\right)$ are known, with the $u_{j}$ on a large regular mesh of $N+1$ points. To evaluate the Fourier coefficients $q_{m}$ for $|m| \leq M$, we search through the $\Phi^{(j)}$ to find those that are closest to the points one would normally use in a discrete Fourier transform, namely the points

$$
\frac{\pi k}{M}, \quad k=0, \ldots, M \text {. }
$$

Denoting those angles by $\Phi_{k}$, and the corresponding values of $q\left(u_{j}\right)$ by $q^{(k)}$, we solve the following linear equations for the Fourier coefficients:

$$
q^{(k)}=\sum_{m=0}^{M} q_{m} \cos m \Phi_{k}, \quad k=0, \ldots, M
$$

We solve this system as follows: if we assume that the function $q(\phi)$ can be expressed exactly as

$$
q(\phi)=\sum_{m=0}^{M} q_{m} \cos m \phi
$$

then we can write $q\left(\Phi_{k}\right)$ in terms of the values $x_{k}=$ $q(\pi k / M)$ as

$$
\begin{aligned}
q\left(\Phi_{k}\right) & =\frac{1}{2 M}\left\{x_{0} \cot \frac{\Phi_{k}}{2} \sin M \Phi_{k}\right. \\
+ & x_{M} \cot \frac{\Phi_{k}-\pi}{2} \sin M\left(\Phi_{k}-\pi\right) \\
& \left.+\sum_{n=1}^{M-1} x_{n} \frac{\sin \Phi_{k}}{\sin \left(\Phi_{k}+\pi k / M\right)} \frac{\sin M\left(\Phi_{k}-\pi k / M\right)}{\sin \left[\left(\Phi_{k}-\pi k / M\right) / 2\right]}\right\}
\end{aligned}
$$

This linear system can then be solved for the $x_{k}$, the function values at the mesh points. The discrete Fourier transform of the $x_{k}$ then gives the coefficients $q_{m}$. The advantage of this is that the system (19) is very well conditioned if the $\Phi_{k}$ are close to the mesh points (16); this is why we chose the mesh points $\Phi_{k}$ as described above.

The system (17) can also be solved as a Vandermonde system. There are $O\left(n^{2}\right)$ direct methods for solving such a system which should work very well [2].

\section{Expressing the Transformation as a Function of $J$}

Let $q_{m}^{(i)}$ and $J^{(i)}$ denote the values of $q_{m}$ and $J$ at $H=$ $H_{i}$, as determined by the procedure just described. To get the required functions of $J$, we invoke the expansions (8) and (9), and determine the coefficients by solving the linear systems

$$
\begin{aligned}
q_{m}^{(i)} & =\sum_{k} q_{m k} B_{k}\left(J^{(i)}\right) \\
H^{(i)} & =\sum_{k} h_{k} C_{k}\left(J^{(i)}\right),
\end{aligned}
$$

where $i=1, \ldots, K$. A possible improvement is to use the values of $H^{\prime}\left(J^{(i)}\right)$ as determined in (12) for an additional constraint on the function $H(J)$. One would then use a larger set of basis functions $C_{k}$, and augment (21) with the additional equations

$$
H^{\prime}\left(J^{(i)}\right)=\sum_{k} h_{k} C_{k}^{\prime}\left(J^{(i)}\right), \quad i=1, \ldots, K
$$

This step should make the whole scheme more selfconsistent, and could be quite worthwhile.

\section{Example}

We have written a code which finds the transformation described for an arbitrary differentiable potential $V$. It computes the transformation from $J=0$ (which is found by finding the minimum of the potential) up through the $J$ corresponding to a given value of $H$. The basis functions $B_{j}$ and $C_{j}$ are both taken to be B-Splines [3] in $\sqrt{J}$, whose knots $t_{i}$ are chosen to be

$$
\begin{aligned}
t_{0} & =\cdots=t_{k-1}=0 \\
t_{i+k} & =\frac{1}{k-1} \sum_{j=i+1}^{i+k-1} \sqrt{J^{(i)}} \quad i=0, \ldots, n-k-1 \\
t_{n} & =\cdots=t_{n+k-1}=\sqrt{J^{(n-1)}}
\end{aligned}
$$

as described on pp. 218-9 of [3]. The code computes $g_{m}$ for $m \leq M$ for a given integer $M$. We do not use the data for $H^{\prime}\left(J^{(i)}\right)$ as described above.

We take as an example the potential $V(q)=1-\cos q$. We know the transformation for this potential:

$$
J=\frac{8}{\pi}\left[\frac{H}{2} K\left(\frac{H}{2}\right)-K\left(\frac{H}{2}\right)+E\left(\frac{H}{2}\right)\right]
$$




$$
\Phi= \begin{cases}\frac{\pi}{2}\left\{\begin{array}{ll}
\left.1-\frac{\left.F\left(\sin ^{-1}[\sqrt{H / 2} \sin q / 2]\right] H / 2\right)}{K(H / 2)}\right\} & p<0 \\
\frac{\pi}{2}
\end{array}\left\{\frac{F\left(\sin ^{-1}[\sqrt{H / 2} \sin q / 2] \mid H / 2\right)}{K(H / 2)}-1\right\}\right. & p>0\end{cases}
$$

Here $F$ and $K$ are elliptic integrals [4].

We will check the accuracy of our transformation by computing $q$ and $H$ on a uniform mesh in $J$ of $10 K$ points and a uniform mesh in $\Phi$ of $10 \mathrm{M}$ points (excluding $\Phi=0$ and $\Phi=\pi)$. First, we compute $H(J)$ at each $J$ mesh point, then substitute that value in $\mathrm{Eq} .(26)$ and compare to the original $J$. We give the maximum value of $\Delta J=\left|J\left(H\left(J_{i}\right)\right)-J_{i}\right| / J_{i}$ in table I. Next, we take $H\left(J_{i}\right)$

\begin{tabular}{|c|c|c|c|c|}
\hline$M$ & $K$ & $\Delta J$ & $\Delta \Phi$ & $\epsilon_{S}$ \\
\hline 4 & 8 & $2 \times 10^{-5}$ & $8 \times 10^{-4}$ & $4 \times 10^{-3}$ \\
4 & 16 & $6 \times 10^{-7}$ & $8 \times 10^{-4}$ & $3 \times 10^{-3}$ \\
4 & 32 & $2 \times 10^{-8}$ & $8 \times 10^{-4}$ & $3 \times 10^{-3}$ \\
4 & 64 & $8 \times 10^{-10}$ & $8 \times 10^{-4}$ & $3 \times 10^{-3}$ \\
4 & 128 & $2 \times 10^{-11}$ & $8 \times 10^{-4}$ & $3 \times 10^{-3}$ \\
\hline 8 & 8 & $2 \times 10^{-5}$ & $8 \times 10^{-4}$ & $2 \times 10^{-2}$ \\
8 & 16 & $6 \times 10^{-7}$ & $4 \times 10^{-5}$ & $9 \times 10^{-4}$ \\
8 & 32 & $2 \times 10^{-8}$ & $2 \times 10^{-6}$ & $3 \times 10^{-5}$ \\
8 & 64 & $8 \times 10^{-10}$ & $8 \times 10^{-7}$ & $7 \times 10^{-6}$ \\
8 & 128 & $2 \times 10^{-11}$ & $8 \times 10^{-7}$ & $7 \times 10^{-6}$ \\
\hline 16 & 8 & $2 \times 10^{-5}$ & $2 \times 10^{-3}$ & $1 \times 10^{-1}$ \\
16 & 16 & $6 \times 10^{-7}$ & $2 \times 10^{-5}$ & $1 \times 10^{-3}$ \\
16 & 32 & $2 \times 10^{-8}$ & $5 \times 10^{-7}$ & $2 \times 10^{-5}$ \\
16 & 64 & $8 \times 10^{-10}$ & $9 \times 10^{-9}$ & $5 \times 10^{-7}$ \\
16 & 128 & $2 \times 10^{-11}$ & $5 \times 10^{-10}$ & $8 \times 10^{-9}$ \\
\hline
\end{tabular}

Table I

Accuracy of the transformation. Quartic B-splines are used throughout. Maximum value of $H$ is 1 .

and $q\left(J_{i}, \Phi_{j}\right)$ on the grid described and compute $\Phi$ using Eq. (27) for each of these values. These results are then compared to the original $\Phi$. We record the maximum value of $\Delta \Phi=\left|\Phi\left(H\left(J_{i}\right), q\left(J_{i}, \Phi_{j}\right)\right)-\Phi_{j}\right|$ in the second column of table I. Finally, we check the symplecticity of the resulting transformation by computing

$$
\epsilon_{S}=\left|\frac{\frac{\partial q}{\partial \Phi} \frac{d H}{d J}}{\sqrt{2[H-V(q)]}}-1\right|
$$

for values where neither the square root nor $\partial q / \partial \Phi$ is zero. The maximum value of this is recorded in the third column of table I.

\section{Conclusion}

We have described a method for determining a transformation of a one-dimensional system described by a Hamiltonian of the form (1) to action-angle variables. A computer program to implement this method has been written, and gives satisfactory results regarding convergence.
We note that this method can be applied even to a $V(q)$ which is only given at a finite number of points $q_{i}$. We simply define $V(q)$ to be a function which passes through these values. Any interpolation method may be used to define such a $V(q)$.

This work was motivated by the desire to give a more thorough treatment of the Vlasov equation for longitudinal instabilities, along the lines followed by Oide and Yokoya [5]. These authors linearize the Vlasov equation about the stationary distribution derived from the Haissinski equation, and then use the action-angle variables $J, \Phi$ of the "distorted potential well" implied by that distribution. The perturbed distribution function $\Psi_{1}(J, \Phi)$ is represented as a Fourier series in $\Phi$ with the coefficients being step functions in $J$. The step function technique has some deficiencies. It gives at best slow convergence as the steps are refined, and makes it difficult to treat the Fokker-Planck term, $-2 \delta(\partial / \partial p)\left(p \Psi_{1}+\partial \Psi_{1} / \partial p\right)$. We think that it would be better to use a $C^{2}$ spline basis for the $J$ dependence of $\Psi_{1}$. Then the Fokker-Planck term can be handled easily with the help of our Fourier series (6) for $q$, since $\partial / \partial p=-(\partial q / \partial J)(\partial / \partial \Phi)+(\partial q / \partial \Phi)(\partial / \partial J)$. Oide's rough treatment of the Fokker-Plancli term by a perturbative method suggests that it is very important in determining thresholds of instabilities.

\section{References}

[1] F. B. Hildebrand, Introduction to Numerical Analysis. New York: McGraw-Hill, 1956.

[2] G. H. Golub and C. F. Van Loan, Matrix Computations. Baltimore: Johns Hopkins University Press, 1989.

[3] C. de Boor, A Practical Guide to Splines. New York: Springer-Verlag, 1978.

[4] M. Abramowitz and I. A. Stegun, eds., Flandbook of Mathematical Functions. New York: Dover Publications, 1972.

[5] K. Oide and K. Yokoya, "Longitudinal single-bunch instability in electron storage rings," Tech. Rep. KEK Preprint 90-10, KEK, April 1990. 
